\title{
Relación entre compromiso laboral y la interacción familia-trabajo en médicos y enfermeros
}

Pablo García Muñoz, Andrés Afonso Martín, Paula Casado García, María Jesús Espejo Tirado y Almudena Gámiz López. Universidad de Granada

Recepción: 7 de junio de 2016 | Revisión: 20 de junio de 2016 | Aceptado: 12 de julio de 2016

Correspondencia: pablogarmu@correo.ugr.es

Citar: Garcia, P., Afonso, A., Casado, P., Espejo, M.J. y Gamiz, A. (2016). Relación entre compromiso laboral y la interacción familiatrabajo en médicos y enfermeros. ReiDoCrea, 5(2), 78-82.

Resumen: El objetivo de la presente investigación es estudiar la relación que existe entre el enriquecimiento/entorpecimiento familia-trabajo y el compromiso laboral en una población de médicos/as y enfermeros/as de nacionalidad española. Para ello se administró a 33 participantes de ambos sexos dos tipos de cuestionarios, uno de ellos es el Utretch Work Engagement Scale (UWES; Schaufeli, Salanova, González y Bakker, 2002) y el otro cuestionario es una versión reducida del Work-Home Interaction-Nijmegen (SWING; Geurts, Taris, Kompier, Dikkers, van Hooff y Kinnunen, 2005). Los resultados obtenidos confirman nuestra hipótesis de que las emociones positivas de la familia hacia el trabajo se asocian de forma positiva con el compromiso laboral. Por lo tanto, el tipo de influencia que ejerce la familia sobre el trabajo puede ser un predictor de variables positivas para éste, es decir, que las emociones positivas que son trasladadas de la familia al trabajo afectan positivamente al compromiso laboral.

Palabras clave: Trabajo | Familia

Relationship Between Work Engagement and Family-Work Interaction in Doctors and Nurses

Abstract: The aim of this research is to study the relationship between the enrichment / torpor work-family and job engagement in a population of doctors and nurses of Spanish nationality. For this was given to 33 participants of both sexes two types of questionnaires, one of them was the Utrecht Work Engagement Scale (UWES; Schaufeli, Salanova, González y Bakker, 2002) and another questionnaire was a reduced version of Work-Home Interaction-Nijmegen (SWING; Geurts, Taris, Kompier, Dikkers, van Hooff y Kinnunen, 2005). The results confirm our hypothesis that positive emotions of the family to work are positively associated with job engagement. Therefore, the type of family influence on the work can be a positive predictor variables for this, it is that positive emotions are transferred from the family to work positively affect the labor commitment.

Keywords: Work | Family

\section{Introducción}

Cualquier organización que dote de importancia y valor a sus recursos humanos comprende la necesidad de evaluar las actitudes de sus trabajadores y de promocionar aquellas que sean positivas para el correcto funcionamiento laboral. Algunos ejemplos de estas actitudes positivas son el compromiso organizacional, la implicación laboral o la satisfacción con el trabajo entre otras. Sin embargo, el presente estudio se centrará en otra actitud: el compromiso laboral, que es definido por Bakker y Schaufeli (2008) como un estado interno de carácter positivo y satisfactorio hacia el trabajo que está determinado por tres componentes: la absorción, el vigor y la dedicación.

Se habla de vigor cuando hay una gran motivación para trabajar con esfuerzo, incluso a pesar de las adversidades. En cuanto a la dedicación, se refiere a una fuerte implicación en el trabajo asociada a emociones positivas (e.g. entusiasmo). Por último, la absorción se caracteriza por una alta concentración en el trabajo que hace tener la sensación de que el tiempo pasa muy rápido, incluso haciendo que no haya deseos de irse a casa (Bakker y Schaufeli, 2008)

Entre las consecuencias positivas que conlleva el compromiso laboral se encuentran la reducción de intención de abandono, una mejora en el desempeño laboral y en la 
salud general, una mayor iniciativa y también mayor motivación para mejorar la formación (Agarwal, Datta, Blake-Beard y Bhargava, 2012; Demerouti, Bakker, Janssen y Schaufeli, 2001; Moreno, Sanz, Rodríguez, y Geurts, 2009; Schaufeli y Salanova, 2008; Schaufeli, Taris y Van Rhenen, 2003; Sonnentag, 2003).

Por otro lado, hoy en día es importante tener en cuenta la conciliación entre la vida familiar y el trabajo en los empleados debido a los efectos negativos que conlleva la falta de equilibrio entre estos ámbitos. Por lo tanto, otra variable relevante en el presente estudio es la interacción familia-trabajo, que se define según Geurts, Taris, Kompier, Dikkers, van Hooff y Kinnunen (2005) como "un proceso en el que el comportamiento de un trabajador en un dominio (por ejemplo, en casa) es influido por determinadas ideas y situaciones (positivas o negativas) que se han construido $y$ vivido en el otro dominio (por ejemplo, en el trabajo)" (p.322). Estudios previos han estudiado esta variable tomando ambas direcciones y centrándose sobre todo en la relación conflictiva (Bakker, Shimazu, Demerouti, Shimada, y Kawakami, 2014; Frone y Rice, 1987). El presente trabajo se centrará en la interacción en la dirección familiatrabajo.

Como ya se ha mencionado anteriormente, estudios previos (Bakker, Shimazu, Demerouti, Shimada, y Kawakami, 2014) ya han estudiado la relación que existe entre el compromiso con el trabajo y la interacción trabajo-familia. Los resultados muestran una correlación positiva entre enriquecimiento familiar y compromiso laboral, aunque sólo en el caso de los hombres y correlaciones no significativas entre la interacción familia-trabajo y el compromiso laboral independientemente del sexo. Rothbard (2001) estudió la relación entre el enriquecimiento familia trabajo y el compromiso laboral, obteniendo como resultado una relación significativa entre ambas variables, siendo mayor para el componente absorción en este caso.

Teniendo esto en cuenta, el objetivo de este estudio es analizar la relación que existe entre el enriquecimiento/entorpecimiento familia-trabajo y el compromiso laboral en una población de médicos/as y enfermeros/as de nacionalidad española y así comparar nuestros resultados con los obtenidos por Rothbard (2001).

Nuestra hipótesis, por tanto, sería analizar si las emociones positivas de la familia hacia el trabajo se asocian de forma positiva con el compromiso laboral.

\section{Método}

\section{Participantes}

Se utilizó un muestreo no probabilístico de conveniencia para obtener la muestra. Dicha muestra se compone de 33 participantes $(M=56,03 D T=5,797)$ de los cuales 13 eran hombres y 19 eran mujeres (un participante no indicó su sexo). En la muestra se cuenta con 16 médicos/as y 14 enfermeros/as del centro de salud de la Zubia y el Hospital Clínico de Granada. Todos participaron de manera voluntaria y gratuita.

\section{Material}

Para medir el compromiso laboral se utilizó la Utretch Work Engagement Scale (UWES) (Schaufeli, Salanova, González y Bakker, 2002), en su versión adaptada a la población española (Salanova, Schaufeli, Llorens, Peiró y Grau, 2000). La escala se compone de 17 ítems que se responden a través de una escala tipo Likert de 7 puntos que va desde 0 (Nunca/Ninguna vez) hasta 6 (Siempre/Todos los días) y que miden los tres componentes de la variable: dedicación, absorción y vigor. La consistencia 
interna de las tres dimensiones de la escala (Salanova, Schaufeli, Llorens, Peiró y Grau, 2000) supera el $\alpha=.70$ recomendado por Nunnaly y Bernstein (1994). En nuestro estudio la consistencia interna de cada escala fue: vigor $(\alpha=.70)$, dedicación $(\alpha=.86)$ y absorción (0.70), por lo que se considera que el instrumento tiene una adecuada fiabilidad.

Para medir la interacción familia-trabajo se utilizó el Survey Work-Home InteractionNijmegen (SWING; Geurts, Taris, Kompier, Dikkers, Van Hooff y Kinnunen, 2005) en su versión reducida en español (Moreno, Sanz, Rodríguez y Geurts, 2009), utilizando solo la escala de interacción familia-trabajo, tanto positiva como negativa. El cuestionario está compuesto por 22 ítems que se subdividen en cuatro subescalas (interacción negativa trabajo-familia y familia-trabajo e interacción positiva trabajofamilia y familia-trabajo) las cuales son respondidas a través de una escala tipo Likert de 4 puntos que va desde 0 (Nunca) hasta 4 (Siempre). La consistencia interna que muestra el instrumento es adecuada, teniendo un alfa que oscila entre .77 y .89 (Moreno, Sanz, Rodríguez y Geurts, 2009). En nuestro estudio la selección de ítems del cuestionario muestra una fiabilidad de .80 .

\section{Procedimiento}

Para la administración de los cuestionarios, se le entregó una copia del cuadernillo a cada participante, siendo completados en el contexto en el que a cada participante le fuese posible (casa o lugar de trabajo). Se pidió que, además de completar las distintas escalas incluidas en el cuadernillo, indicaran datos sociodemográficos tales como su sexo, edad y puesto de trabajo. Una vez completados los cuadernillos se les agradeció su participación y se les explicó el objetivo del estudio.

\section{Análisis de datos}

Para el análisis de datos se ha realiza una correlación entre variables a través del programa informático estadístico SPSS en su versión 22.0.

\section{Resultados}

Como se muestra en la tabla 1 , se observa la existencia de una correlación positiva entre las puntuaciones de compromiso laboral y la interacción positiva familia-trabajo $(r=.493)$. Por otro lado, se ha obtenido una correlación positiva entre la interacción positiva familia-trabajo y las dimensiones dedicación $(r=.560)$ y absorción $(r=.436)$, obteniendo en dedicación un nivel de significación bilateral de, 01 , mientras en absorción lo hace de la misma forma pero a un nivel de significación de ,05 bilateral. En cambio, se ha hallado una correlación negativa entre la dimensión de vigor del compromiso laboral y la interacción negativa familia-trabajo $(r=.344)$ a un nivel de significación de, 05 .

\begin{tabular}{|ccccccc|}
\hline \multicolumn{6}{|c|}{ Tabla 1. Correlaciones entre las variables relacionadas con el compromiso familiar y la interacción familia-trabajo } \\
\hline & Vigor & Dedicación & Absorción & Compromiso & Enriquecimiento F-T & Conflicto F-T \\
\hline Vigor & - & $.647^{* *}$ & $.629^{* *}$ & $.835^{* *}$ & .302 & $-.344^{*}$ \\
Dedicación & - & $.773^{* *}$ & $.913^{* *}$ & $.560^{* *}$ & -.137 \\
Absorción & & & - & $.914^{* *}$ & $.436^{*}$ & -.156 \\
Compromiso & & & - & $.493^{* *}$ & -.230 \\
Enriquecimiento F-T & & & & - & .189 \\
Conflicto F-T & & & & - \\
\hline Nota: ${ }^{*} p<0,05 ;{ }^{* *} p<0,01$ & & & & \\
\hline
\end{tabular}




\section{Discusión}

Tras el análisis de los resultados obtenidos, se confirma la hipótesis inicial, donde las puntuaciones obtenidas en la escala positiva de interacción familia-trabajo de la escala SWING (2005) correlacionan positivamente con las puntuaciones obtenidas en compromiso laboral. Esto podría interpretarse indicando que las emociones positivas que se trasladan de la familia al trabajo afectan positivamente al compromiso laboral.

Otro dato interesante son las relaciones positivas halladas entre enriquecimiento familia-trabajo y los componentes dedicación y absorción. Estos datos podrían ser indicativos de la posible mejora en la calidad de la relación familia-trabajo promocionando conductas positivas como estar atento o promocionando sentimientos positivos mientras se trabaja. Por otra parte, observamos diferencia con respecto a los resultados obtenidos en Rothbard (2001), donde la relación significativa entre familiatrabajo y compromiso era mayor en el ámbito de absorción, mientras que nuestros resultados muestran una relación positiva y significativa también en el ámbito de dedicación.

Por otro lado, la correlación negativa podría indicar que cuanto más conflicto haya en la conciliación de la familia con el trabajo menos vigor tendrá el sanitario, es decir, habrá menos motivación para trabajar con esfuerzo a pesar de las adversidades. Este es un dato importante dado que el personal sanitario tiene un trabajo en bastantes ocasiones duro y con dificultades. Debido a esto, lo esperable sería encontrarse con altos niveles de vigor.

Por tanto, el tipo de influencia que ejerce la familia sobre el trabajo puede ser un predictor de variables positivas para el trabajo como la reducción en la intención de abandono, un mejor rendimiento laboral o mayor motivación para seguir formándose (Agarwal et al., 2012; Demerouti et al., 2001; Moreno et al., 2009; Schaufeli et al., 2008; Schaufeli et al., 2003; Sonnentag, 2003), pudiendo vislumbrarse el compromiso laboral como una variable mediadora entre la influencia de la familia en el trabajo y las variables positivas citadas anteriormente, aunque según los datos no es posible afirmar que exista esta relación.

Estos resultados muestran la posibilidad de poder influir tanto en una variable como en la otra a través de las estrategias convenientes para promocionar conductas positivas para el trabajo. No cabe duda de la importancia en el ámbito sanitario de un buen rendimiento, una buena salud o ganas de seguir formándose para ofrecer el mejor servicio a los pacientes pero estos resultados podrían aplicarse en otro tipo de trabajadores de distintas empresas. Por tanto, investigaciones futuras podrían ir encaminadas a corroborar si estos mismos resultados se dan en trabajadores de distintos ámbitos.

Por último hay que mencionar algunas limitaciones presentes en este estudio, entre las que se encuentra sobre todo el tamaño de la muestra. Para estudios posteriores podría aumentarse el tamaño de la muestra y obtener la participación de trabajadores sanitarios de distintos centros de salud y hospitales de la región e incluso de toda la comunidad autónoma. 


\section{Referencias}

Agarwal U., Datta, S., Blake-Beard, S., \& Bhargava, S. (2012). Linking LMX, innovative work behaviour and turnover intentions. The mediating role of work engagement. Career Development International, 17(3), 208-230.

Bakker, A.B., \& Schaufeli, W.B. (2008). Positive organizational behavior: engaged employees in flourishing organizations. Journal of Organizational Behavior, 29, 147-154.

Bakker, A. Shimazu, A., Demrouti, E., Shimada, K., \& Kawakami, N. (2014). Work engagement versus workaholism: a test of the spillovercrossover model. Journal of Managerial Psychology 29(1), 63-80.

Clark, M., Michel, J., Stevens, G., Howell, J., \& Scruggs, R. (2013). Workaholism, Work Engagement and Work-Home Outcomes: Exploring the Mediating Role of Positive and Negative Emotions. Stress Health, 30, 287-300.

Demerouti, E., Bakker, A.B., Janssen, P.P.M. \& Schaufeli, W.B. (2001). Burnout and engagement at work as a function of demands and control. Scandinavian Journal of Work, Environment \& Health, 27, 279-286.

Frone, M.R., \& Rice, R.W. (1987). Work-family conflict: The effect of job and family involvement. Journal of Occupational Behavior, 8(1), 45-53.

Geurts, S., Taris, T.W., Kompier, M.A.J., Dikkers, J.S.E., Van Hooff, M.L.M., \& Kinnunen, U.M. (2005). Work-home interaction from a work psychological perspective: Development and validation of a new questionnaire, the SWING. Work \& Stress, 19(4), 319-339.

Greenhaus, J.H., \& Powell, G. (2006). When work and family are allies: A theory of work-family enrichment. Academy of Management Review, 31(1), 72-92.

Moreno, B., Sanz, A. I., Rodríguez, A. y Geurts, S. (2009). Propiedades psicométricas de la versión española del Cuestionario de Interacción Trabajo-Familia (SWING). Psicothema, 21(2), 331-337.

Nunnaly, J.C., \& Bernstein, I.H. (1994). Psychometric theory. New York, NY: McGraw-Hill.

Oramas, A., Marrero, I., Cepero, E., del Castillo, N.P., \& Vergara, A. (2014). Escala de Work engagement de Utrech. Evaluación del work engagement en trabajadores cubanos. Revista cubana de salud y trabajo, 15(2), 47-56.

Rothbard, N.P. (2001). Enriching or depleting? The dynamics of engagement in work and family roles. Administrative Science Quarterly, 46(4), $655-84$.

Runhaar, P., Konermann, J., \& Sanders, K. (2013). Teachers' organizational citizenship behaviour: Considering the roles of their work engagement, autonomy and leader-member exchange. Teaching and teacher education, 30, 99-108.

Salanova, M., Schaufeli, W.B., Llorens, S., Peiró, J.M. y Grau, R. (2000). Desde el "burnout" al "engagement": ¿una nueva perspectiva? Revista de Psicología del Trabajo y de las Organizaciones, 16(2), 117-134

Schaufeli, W.B., Salanova, M., González-Roma. V., \& Bakker, A.B. (2002a). The measurement of engagement and burnout and: A confirmative analytic approach. Journal of Happiness Studies, 3, 71-92.

Schaufeli, W.B., Taris, T.W., \& Van Rhenen, W. (2008). Workaholism, burnout and engagement: Three of a kind or three different kinds of employee well-being? Applied Psychology: An International Review, 57(2), 173-203.

Schaufeli, W. B., \& Salanova, M. (2008). Enhancing work engagement through the management of human resources. In K. Nåswall, M. Sverke, \& J. Hellgren (Eds.), The individual in the changing working life (pp. 380-404). Cambridge: Cambridge University Press.

Sonnentag, S. (2003). Recovery, work engagement, and proactive behavior (2003): A new Look at the interface between non-work and work. Journal of Applied Psychology, 88, 518-528.

Strauser, D.R., O'Sullivan, D., \& Wong, A. (2012). Work personality, work engagement, and academic effort in a group of college students. Journal of Employment Counseling, 49(2), 50-61. 